\title{
Junior doctors are performing fewer emergency assessments - a cause for concern
}

\author{
Linda Waddell, ${ }^{1}$ Colin Crawford ${ }^{2}$
}

The Psychiatrist (2010), 34, 268-270, doi: 10.1192/pb.bp.109.026013

${ }^{1}$ Hairmyres Hospital, Lanarkshire: ${ }^{2}$ Falkirk and District Royal Infirmary, Scotland, UK

Correspondence to Linda Waddell (linda.waddell@nhs.net)

\begin{abstract}
Aims and method Due to concerns regarding the reduced exposure of junior trainees to risk assessment, we have examined emergency assessments carried out in Forth Valley, Scotland, during a 4-month period to ascertain the assessor, time of assessment and outcome.
\end{abstract}

Results During the 4 months of the audit, an average of 13 emergency psychiatric assessments were carried out by each trainee. The majority of these assessments occurred overnight (81\%).

Clinical implications Experience of emergency assessments by trainees was limited and tended to occur during on-call periods when there is little chance for teaching. With this limited exposure, trainees are missing out on valuable experience in emergency risk assessment and management planning.

Declaration of interest None.
With the advent of Modernising Medical Careers and the European Working Time Directive, many trusts have made changes to service provision in order to deal with reducing junior doctor hours. Targets set out in The NHS Plan ${ }^{1}$ and Delivering for Mental Health ${ }^{2}$ regarding waiting times and mental health service provision have been a major factor in changes to mental health services, including the creation of new specialist teams and nurse-led services. As regards service provision, specialist nurses have been shown to reduce waiting times and improve capacity. ${ }^{3}$ It has also been shown that assessments following self-poisoning have similar outcomes regardless of whether they are carried out by a liaison nurse or trainee psychiatrist, ${ }^{4}$ lending weight to the argument that nurses can carry out roles previously considered the staple of the on-call junior psychiatrist. On the other hand, these changes have resulted in essential tasks, for example risk assessment following suicide attempt, being less frequently carried out by junior doctors, thus reducing vital clinical experience for the junior trainee. ${ }^{3}$ Junior doctors also have less time to experience psychiatry, with 4-month rotations of foundation year 2 (FY2) and general practice (GP) trainees now the norm.

The Royal College of Psychiatrists has voiced concern about the opportunity of junior psychiatrists to carry out emergency psychiatric assessments. ${ }^{5}$ The College has recommended 'a minimum of 55 nights on call during the period of basic specialist training' and assessment of at least 50 individuals 'with a range of diagnosed conditions and with first line management plans conceived and implemented'. ${ }^{6}$ The GP curriculum includes the need to attain competence in suicide risk assessment (www.rcgp-curriculum.org.uk), which in turn necessitates the opportunity to carry out emergency psychiatric assessments. Historically, the majority of emergency psychiatric assessments carried out by the junior doctor have occurred when on call, and senior house officers agreed that on-call shifts offer an important learning experience. ${ }^{7}$ With the evolution of 24-hour crisis teams and the increase in GP and accident and emergency referrals being assessed out of hours by psychiatric nurses, this no longer seems to be the case.

In 2005, an area-wide intensive home treatment team (IHTT) was set up in Forth Valley, Scotland, with the aim of treating more patients at home, reducing admissions and bed occupancy. A major catalyst for the introduction of the new team was the new Mental Health (Care and Treatment) (Scotland) Act 2003 which advocated the least restrictive measures being used to treat individuals with mental disorder. The team operates with extended hours, 7 days a week, but is not a 24-hour service. Referrals for emergency assessment are received from a number of sources, including GPs, community mental health teams and the liaison psychiatry team. Before IHTT was established, all such referrals would have been received by the duty doctor on call for the day and assessed by them alone on the ward. Now, virtually all referrals go through IHTT with the majority of individuals being assessed by nursing staff. Consequently, the role of the daytime duty doctor has changed greatly and concerns are raised regarding the reduced exposure of junior doctors to first-line emergency assessments, in particular the opportunity to carry out a competent risk assessment, formulate an appropriate management plan and decide the need for admission. The duty doctor on call during the day is encouraged to contact the IHTT if their on-call duties allow them time to assess 
referrals with the team. Outwith IHTT hours the emergency assessments are carried out by the duty doctor allowing first-line exposure to emergency psychiatry.

The purpose of this paper was to examine the number of emergency assessments carried out by junior doctors in psychiatry working in Forth Valley during a 4-month period, to ascertain when the majority of these assessments were occurring and what their outcome was.

\section{Method}

Thirteen junior doctors (FY2, GP trainee and psychiatry specialty trainees years 1-3) work a full shift to cover the two acute psychiatric sites in Forth Valley. One doctor is on duty from $09.00 \mathrm{~h}$ to $21.00 \mathrm{~h}$ weekdays and then hands over to the resident on-call night duty doctor covering until $09.00 \mathrm{~h}$ the following morning. Two junior doctors work day shifts at the weekend, one covering each site. The total population served is nearly 300000 and covers an area of over 1000 square miles.

Detailed records of referral information and outcome for emergency assessments have been kept since the introduction of the IHTT. This information was retrospectively examined to gather details on the number of assessments being carried out by junior doctors, their time, outcome, and whether the assessment was carried out alone or in conjunction with IHTT staff. Data were examined during a 4-month rotation block from August to December 2008 allowing the experience garnered by GP and FY2 trainees during their entire psychiatry block to be examined as well as giving an indication of the experience gained by the psychiatry trainees during the rotation period. The junior doctor rota was compared with the dates of assessments to ascertain the average number of emergency assessments carried out by each individual doctor.

\section{Results}

Over the 4-month period, 168 (83\%) emergency assessments were carried out by IHTT staff without junior doctor input, 15 (7\%) with junior doctor co-assessing and 19 (9\%) by the junior doctor alone during IHTT working hours. In addition to these 202,80 patients requiring clerking were also sent to the ward during this time for the duty doctor to see but the decisions regarding risk and the need for admission had already been made by the referrer, who in most cases was a senior psychiatrist or liaison nurse (following discussion with the liaison consultant). As suspected, the majority of emergency assessments carried out by junior trainees were conducted during nights on call $(n=149)$ : 83 individuals (56\%) were admitted and 66 (44\%) sent home, with outpatient follow-up in the majority of cases. The outcome of all emergency assessments is shown in Table 1.

On average, each junior doctor carried out 1 assessment in conjunction with a member of the IHTT (range 0-3), 1 lone assessment on the day shift (range 0-4), and 11 assessments during nights on call (range 7-16); each junior doctor conducted on average 13 emergency psychiatric assessments.

\section{Discussion}

Exposure to emergency psychiatry and experience carrying out risk assessments and formulating management plans

\begin{tabular}{|c|c|c|}
\hline \multirow[t]{3}{*}{ Table 1} & $\begin{array}{l}\text { s in Forth } \\
\text { the audit }\end{array}$ & ley over \\
\hline & \multicolumn{2}{|c|}{ Referred individuals, $n(\%)$} \\
\hline & Admitted & Not admitted \\
\hline IHTT staff & $17(10)$ & $151(90)$ \\
\hline IHTT staff + junior doctor & $4(27)$ & $11(73)$ \\
\hline Junior doctor alone, day & $12(63)$ & $7(37)$ \\
\hline Junior doctor alone, night & $83(56)$ & $66(44)$ \\
\hline
\end{tabular}

IHTT, intensive home treatment team.

are essential for doctors planning a career in psychiatry. It is also desirable for GP trainees to attain these skills to a level of proficiency that will enable them to feel confident in assessing when referral to psychiatry is appropriate and when it is not. Currently, junior doctors in Forth Valley are getting almost all their emergency psychiatry experience when on call overnight. Unfortunately, this is the period with the least back-up from colleagues, other health professionals and seniors and the prospect of any teaching from seniors is non-existent. Despite the fact that junior doctors are encouraged to discuss during supervision interesting cases seen on call, the immediacy of the case and the reality of making a decision in real time is no longer there.

Because of concerns regarding reduced juniors' experience at first-line assessments, the Royal College of Psychiatrists advises that trainees are regularly rostered to obtain this clinical experience under supervision'. ${ }^{6}$ In Forth Valley there is one junior trainee attached to the team for half of the week but given his commitments in the other half of the job there was little opportunity for him to spend time with IHTT. During the 4 months audited the trainee carried out the same number of assessments as other juniors not attached to the team. The duty doctor on each day is also encouraged to do assessments with team members if they have free time between on-call duties. This clearly did not occur very often during the audit as only one assessment per trainee was carried out with IHTT. On-call work is unpredictable and likely to result in trainees taking their breaks during quieter periods in the day and therefore being less likely to volunteer for extra work with the IHTT. Only highly motivated individuals are likely to volunteer to do joint assessments. The number of overall emergency assessments done by juniors currently is low (average 13). There is a possibility of the team moving to a 24-hour service in the future, thus further reducing the experience the junior trainees are able to get. One possible way of combating this would be to have junior doctors rotate to do a period of time working full time with the IHTT. This could be a week-long block where they leave their usual post and work full time in the team, just as junior medics spend week-long periods in the acute receiving medical ward. Alternatively, placing one trainee per rotation full-time in the team would allow a number of psychiatry trainees to gain experience of working in the team in their junior trainee years. Unfortunately, service commitments elsewhere would be the main reason that this is unlikely to happen as the team functions well without a 
junior doctor and their presence would be purely for training purposes. Emergency risk assessment and management planning skills could also be attained by junior trainees working within the liaison psychiatry service, which is already a common practice in many areas of the UK.

There have been debates about the merits of developing specialist teams such as the IHTT. ${ }^{8}$ Despite these reservations there has been a huge expansion of crisis teams particularly in England and similar services are now emerging in Scotland. The evidence points to a reduction in admissions and shorter length of stay. ${ }^{9}$ In Forth Valley this has allowed a $20 \%$ reduction in acute beds while keeping bed occupancy at $80 \%$ with the remaining beds. ${ }^{10}$ In areas with 24-hour crisis services the only way that junior trainees will get meaningful experience in emergency assessments is with a formal placement with the service. This may be of benefit to psychiatry trainees but is unlikely to be possible for other trainees with such short rotations. More creative solutions are going to be needed to fill this gap.

\section{About the authors}

Linda Waddell is ST6 working in Hairmyres Hospital, Lanarkshire, and Colin Crawford is Consultant Psychiatrist, Intensive Home Treatment Team, Falkirk and District Royal Infirmary, Scotland, UK.

\section{References}

1 Department of Health. The NHS Plan: A Plan for Investment, a Plan for Reform. Department of Health, 2000.

2 Scottish Executive. Delivering for Mental Health. Scottish Executive, 2006 (http://www.scotland.gov.uk/Resource/Doc/157157/0042281. pdf).

3 Woodall AA, Roberts S, Slegg GP, Menkes DB. Emergency psychiatric assessments: implications for senior house officer training. Psychiatr Bull 2006; 30: 220-2.

4 Griffin G, Bisson Jl. Introducing a nurse-led deliberate self-harm assessment service. Psychiatr Bull 2001; 25: 212-4.

5 Royal College of Psychiatrists Psychiatric Trainees Committee. Finding the Balance: The Psychiatric Training Value of Out of Hours Working. Royal College of Psychiatrists, 2008 (http://www.rcpsych.ac.uk/pdf/ PTC\%20The\%20training\%20value\%20of\%2000H.pdf).

6 Royal College of Psychiatrists. Specialist Training in Psychiatry: A Comprehensive Guide to Training and Assessment in the UK for Trainees and Local Educational Providers (OP69). Royal College of Psychiatrists, 2009.

7 Callaghan R, Hanna G, Brown N, Vassilas C. On call: valuable training experience for senior house officers? Psychiatr Bull 2005; 29: 59-61.

8 Pelosi AJ, Jackson GA. Home treatment - enigmas and fantasies. BMJ 2000; 320: 308-9.

9 Glover G, Arts G, Babu KS. Crisis resolution/home treatment teams and psychiatric admission rates in England. Br J Psychiatry 2006; 189: 441-5.

10 Coutts P, McLaren G, Crawford C. Providing alternatives to inpatient care: the intensive home treatment team pilot in Forth Valley. Ment Health Rev J 2006; 11: 37-40.

\title{
Liaison psychiatry services in south England
}

\author{
Jackie Gordon, ${ }^{1}$ Sonia Wolf ${ }^{1}$
}

The Psychiatrist (2010), 34, 270-273, doi: 10.1192/pb.bp.109.026468

${ }^{1}$ Mental Health Liaison Team, Worthing Hospital, UK

Correspondence to Jackie Gordon (jackie.gordon@

sussexpartnership.nhs.uk)
Aims and method To investigate liaison psychiatry services across 38 acute trusts in the south of England. We used a telephone survey and compared the results to service structure and function as recommended by the Royal College of Physicians and the Royal College of Psychiatrists.

Results Approximately two-thirds of trusts surveyed had a dedicated liaison service and this was not significantly related to hospital size. Most liaison teams were understaffed in all disciplines and only a third had a full-time consultant. Services for specialist patient groups were generally well provided for; $37 \%$ of teams had been created in the past 5 years and 33\% were planning to increase their staffing levels in future.

Clinical implications Liaison services in the south of England are similar to those in other parts of the UK that have been surveyed. Although the services did not meet the Colleges' recommendations, our study shows some recent growth and development in this specialty.

Declaration of interest None.
Liaison psychiatry service provision has been shown to be variable, despite clear guidance from the Royal College of Physicians and the Royal College of Psychiatrists. ${ }^{1}$ Previous work in London, north-west England and Wales has shown inadequate staffing and wide variation in availability of services. $^{2-5}$ In 2008, the Academy of Medical Royal Colleges (AMRC) recommended a 24-hour mental health liaison service, specifying a timely first-line attendance and 